\section{$\underset{\substack{\text { hommes } \\ \text { \& migrations }}}{ }$}

\section{Hommes \& migrations}

Revue française de référence sur les dynamiques

migratoires

$1284 \mid 2010$

Migrations et environnement

\title{
Les oasis du Drâa au Maroc
}

Rupture des équilibres environnementaux et stratégies migratoires

\section{Mohamed Aït Hamza, B. El Faskaoui et Alfons Fermin}

\section{OpenEdition \\ 1 Journals}

\section{Édition électronique}

URL : http://journals.openedition.org/hommesmigrations/1241

DOI : 10.4000/hommesmigrations. 1241

ISSN : 2262-3353

Éditeur

Musée national de l'histoire de l'immigration

Édition imprimée

Date de publication : 1 mars 2010

Pagination : 56-69

ISSN : 1142-852X

Référence électronique

Mohamed Aït Hamza, B. El Faskaoui et Alfons Fermin, "Les oasis du Drâa au Maroc », Hommes \&

migrations [En ligne], 1284 | 2010, mis en ligne le 01 mars 2012, consulté le 17 juin 2020. URL : http:// journals.openedition.org/hommesmigrations/1241 ; DOI : https://doi.org/10.4000/

hommesmigrations. 1241 


\section{Les oasis du Drâa au Maroc Rupture des équilibres environnementaux et stratégies migratoires}

Par M. Aït Hamza, géographe, directeur du Centre des études historiques et environnementales (CEHE) à l'Institut royal de la culture amazighe (IRCAM), Rabat B. El Faskaoui, professeur de géographie, Université Moulay Ismail, Meknès, Maroc et Alfons Fermin, chercheur à faculté des sciences sociales de l'Université Erasme de Rotterdam lors du projet EACH-FOR

La mobilité horizontale des populations est souvent conçue comme une réaction à des déséquilibres socio-économiques, spatiaux ou politiques. Mais la part des facteurs environnementaux reste à définir.

Car les modifications de l'environnement suscitent tout autant le désir de partir que des stratégies inédites d'adaptation. Pour comprendre leur poids dans l'évolution du rapport entre un territoire et ses habitants, des investigations empiriques ont été menées dans la province de Zagora, au sud du Maroc. Aux confins sahariens du pays, enquête dans deux palmeraies de la vallée du Drâa : M’hamid et Ktaoua. 
Situé au sud de la chaîne de l'Atlas, le bassin du Draâ s'étale sur une longueur d'environ $1200 \mathrm{Km}$, entre les sommets du Haut Atlas calcaire qui culmine à plus de 4000 m d'altitude et l'embouchure de l'oued sur l'océan Atlantique. Les crêtes qui le constituent, dans sa partie amont, prennent la direction nord-est/sud-ouest, avant de laisser la place aux larges hamadas après le Jbel Bani. Le lit de l'oued, formé d'un chapelet de palmeraies successives, larges de moins de $5 \mathrm{~km}$ (Mezguita, Tinzouline, Ternata, Fezouata, Ktaoua et M'hamid), constitue l'espace où se concentrent les activités humaines et les habitations ( $\left.\operatorname{carte} \mathrm{n}^{\circ} 1\right)$.

Ktaoua et M'hamid ${ }^{(1)}$, situées à la lisière du Sahara, sur les confins algéro-marocains, constituent les oasis les plus méridionales de la moyenne vallée du Drâa, les plus menacées par les risques environnementaux (sécheresse, salinité, ensablement, Bayoud, invasions acridiennes...), mais aussi les plus enclavées.

Largement ouvertes sur les

\section{Carte $n^{\circ} 1$ : croquis de situation}

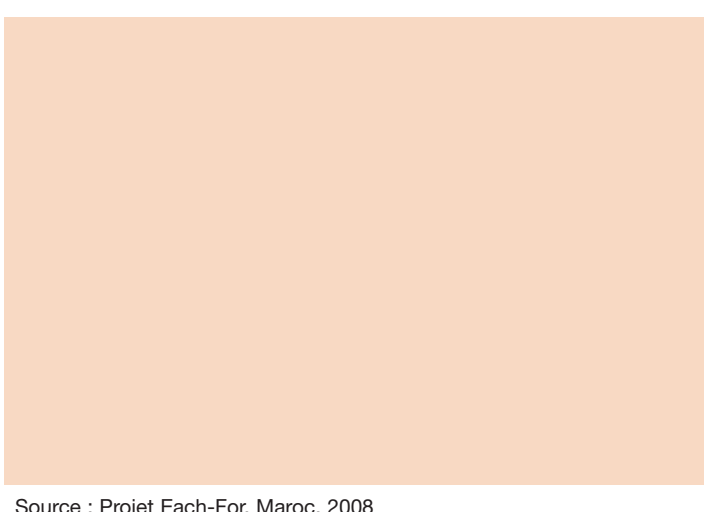

Source : Projet Each-For, Maroc, 2008

influences sahariennes, ces oasis constituent un don des eaux superficielles venant essentiellement du Haut Atlas central. Déterminé par ces conditions et par la continentalité, leur climat se caractérise par ses fortes températures notamment entre juin et septembre (43 à 50 $)$; ses fortes amplitudes thermiques ( $20^{\circ}$ à Tagounite); la très longue durée des heures d'insolation (entre 3055 et 3078 heures/an); la forte évapotranspiration notamment en été ; la rareté et la très forte variabilité annuelle et interannuelle des précipitations.

\section{La vallée du Drâa, un cadre socio-spatial contraignant}

La station de Tagounite, située dans la palmeraie de Ktaoua, enregistre les écarts les plus importants (entre 4 et $150 \mathrm{~mm}$ ) avec une très grande fréquence des années en dessous de la moyenne (15 sur 26). Les épisodes secs se caractérisent par leur profondeur et leur fréquence. Les pluies tombent souvent en automne et en février sous forme d'averses concentrées et agressives. Les fortes inondations qui en résultent 
érodent, annuellement, les terrasses de cultures aménagées le long des cours d'eau. La forte évapotranspiration due à la fréquence des tempêtes, à la rareté du couvert végétal et aux fortes chaleurs qui sévissent sur la zone presque toute l'année, entraîne un desséchement brutal de l'air et un accroissement constant des besoins en eau d'irrigation. La rareté et l'irrégularité des pluies, outre l'absence quasi totale des eaux de surface, forment les éléments les plus structurants et les plus déterminants des installations humaines. Les conflits autour de l'eau et des pâturages constituent le fond de la toile historique de ces oasis.

La vallée du Drâa, ancienne route caravanière entre le Sahara et le nord du Maroc, exerçait durant sa longue histoire un important attrait sur des populations venues du sud comme du nord. Il en a résulté un brassage ethnique considérable. Outre les Draouas $^{(2)}$ (population noire), supposés être les autochtones, la population de la vallée se composait des Arabes venus du Sahara, de tribus venues du nord (Aït Sedrate ${ }^{(3)}$, des anciens nomades (Aït Atta ${ }^{(4)}$ ), de communautés à caractère sacro-saint (Chorfas et Morabitines) et de Juifs ${ }^{(5)}$.

De cette mosaïque, la vallée a hérité une structure socio-ethnique très diversifiée et très hiérarchisée. Ce brassage, souvent effectué dans des conditions conflictuelles, entre dominants et dominés ${ }^{(6)}$, anciens et nouveaux arrivants, a marqué la mémoire collective, malgré le laminage sous l'effet de l'école, de l'exode, de la migration, du tourisme et de l'urbanisation croissante.

\section{Faiblesse des ressources et accroissement des besoins}

Outre le taux d'accroissement naturel, toujours fort ${ }^{(7)}$, le dynamisme démographique des oasis du Drâa en général, et des deux palmeraies de Ktaoua et M'hamid en particulier, s'explique par divers facteurs : le conflit frontalier entre le Maroc et l'Algérie qui perdure depuis 1963 ; la sédentarisation accélérée des nomades, en partie due aux effets du conflit et aux sécheresses (370 ménages en 1982 et seulement 141 en 1994); le départ de la communauté juive dès la fin des années cinquante ; la récente attraction qu'exercent les déserts sur les touristes (chasse, sport, festival des nomades, musique des déserts....).La régulation qu'exerce la migration provoque la régression de la population malgré son fort accroissement naturel. Entre 1994 et 2004, la population des deux palmeraies a enregistré une nette régression $(0,1 \%$ pour Ktaoua et $0,9 \%$ pour M'hamid) comparé à la province $(0,9 \%)$ et à l'espace national $(1,4 \%)$. En fait, entre 1971 et 2004, les deux palmeraies ont perdu un effectif de 3666 habitants, soit environ 111 personnes par an. La palmeraie de M'hamid a perdu 288 ména- 
ges entre 1960 et 2004 et celle de Ktaoua, 795. Si on ne considère que la population rurale, entre 1982 et 2004, M'hamid est passée de 8671 habitants à 7764 et Ktaoua de 24780 à seulement 17 553, soit une perte respectivement de 907 et 7227 habitants. Mais le taux des jeunes de moins de 15 ans reste très élevé (40\%) comparé au reste du pays (31,2\%). C'est dire combien le poids des besoins en termes d'équipements sociaux et d'emplois s'avère très lourd à un moment où le taux d'analphabétisme atteint presque $49 \%$, le taux des actifs n'excédant pas les 20,5\%. La forte régression de la population rurale est donc à mettre en relation avec les sécheresses aiguës qu'a connues le Maroc notamment au début des années quatre-vingt.

La population oasienne, depuis des temps immémoriaux, a développé des formes d'adaptation à son environnement. La ges-

Très rare, l'eau, facteur structurant et déterminant, est soumise à un partage très rigoureux. Les parts de chacun sont déterminées en fonction de son statut socio-économique. tion de la rareté et de la fragilité des ressources agraires et pastorales est une des manifestations de ce génie civilisationnel. Dans cet esprit, l'espace oasien que constituent les palmeraies de Ktaoua et M'hamid est formé de deux grandes parties économiquement et statutairement différenciées : la partie cultivable, totalement privatisée et la partie réservée à l'élevage extensif, constituée de parcours collectifs.

\section{Le défi de l'eau au cœur de la survie d'un territoire}

Les terres agricoles qui ne représentent que moins de $1 \%$ de la surface totale se limitent aux lits des oueds. Très rare, l'eau, facteur structurant et déterminant, est soumise à un partage très rigoureux. Les parts de chacun sont déterminées en fonction de son statut socio-économique. Les droits d'eau, s'ils ne sont pas affiliés à une institution religieuse (mosquée ou zaouia), s'alignent sur la propriété de la terre. Ils déterminent, outre la position socio-économique du propriétaire, toutes ses stratégies d'adaptation. La gestion communautaire de l'eau est un élément fondamental de cette civilisation. Ainsi, malgré la construction d'un grand barrage, le statut de l'eau, de la terre et des arbres continue à paralyser les structures de production. La micropropriété règne. Plus de 80 \% des paysans ont moins d'un hectare de terres. Lémiettement des champs et leur dispersion forment le caractère dominant (entre 1 et 8 parcelles par exploitation). Les rares exploitations ayant encore une taille 
respectable sont celles nouvellement conquises sur le collectif par le biais des partages et du pompage ou celles soumises au régime de l'indivision.

Les arbres, notamment les palmiers dattiers, au vu de leur importance économique et sociale, peuvent aussi constituer un titre foncier à part entière et sujet à des transactions.

Les techniques de production sont restées, dans leur ensemble, traditionnelles. La mécanisation est rare, eu égard à l'étroitesse des propriétés, à l'éparpillement des parcelles et aux arbres peu nombreux. Au-dessous des divers arbres pousse une multitude de cultures saisonnières. Seul le henné exige des espaces dégagés et des traitements spéciaux ${ }^{(8)}$.

Dans un tel contexte de rareté et de déterminisme naturel, seuls les dattes et le henné rentrent dans un circuit d'échange local et national. Les oasiens, pour subsister, gèrent communément leur patrimoine en jouant jadis sur la diversification des produits et la complémentarité des terroirs et aujourd'hui sur la migration et la diversification des activités et des ressources. Dans ce cadre, la pratique de l'élevage est considérée comme un élément d'adaptation économique et écologique stratégique. En fait l'élevage, associé à l'agriculture, permet de réaliser la complémentarité des terroirs et des modes de vie. Il permet de développer un capital facilement mobilisable et modulable en cas de crise ou de nécessité socio-économique. La diversité 
des espèces animales (camelins, ovins, caprins) peut être aussi expliquée par ce souci de contourner le risque qui guette la production.

La race ovine (Dmane), à la forte capacité prolifératrice, constitue une spécificité et un atout de ces oasis (25 329 têtes). Les caprins, plus rustiques, font plutôt une spécialité de transhumants (28 329 têtes). Les camelins, espèce purement saharienne (5 062 têtes), outre leur capacité de transport, restent un symbole de richesse et de conquête. Le cheptel symbolise, outre l'aisance, l'indépendance, la capacité de conquérir et de défendre un territoire tribal collectif. Avec la colonisation, l'installation de l'administration moderne, le recul du pouvoir communautaire, la fixation imposée aux différentes tribus, l'apparition des centres urbains et, avec eux, la multiplication des activités non agricoles, l'élevage a beaucoup régressé. Les Ait Atta ${ }^{(9)}$ et Aribs, grands éleveurs de caprins et de camélidés, ont été les premiers à être victimes de ces transformations.

\section{La rupture des équilibres environnementaux}

Eu égard aux conditions naturelles décrites ci-dessus, le débit moyen de Oued Drâa à Zagora est de 13,4 m3/s, le maximum enregistré en 1965 atteint $213 \mathrm{~m} 3 / \mathrm{s}$ contre seulement $0,13 \mathrm{~m} 3 / \mathrm{s}$ en août de la même année. Ainsi, parallèlement à la rareté et à l'irrégularité de plus en plus accentuées des eaux, leur salinité augmente. De nombreux fléaux dévastent les oasis : l'appauvrissement des sols, la perte du couvert végétal, l'ensablement, et par conséquent la régression des rendements.

Afin de remédier, en partie, à ces problèmes, l'État a édifié le barrage d'El Mansour Eddahbi ${ }^{(10)}$ en 1972. Conçu pour endiguer les crues, réguler l'écoulement outre la production de l'électricité et l'alimentation des centres urbains en eau potable, ce barrage a généré des effets pervers en perturbant l'alimentation de la nappe, en arrêtant la fertilisation naturelle des sols et en asséchant le lac d'Iriqi, jadis fréquenté 
par une importante faune et par des nomades. Cette perturbation du système écologique, déjà fragile, a renforcé la tendance à la désertification.

L'avancement du désert est une réponse naturelle qui ne s'est pas fait attendre. Les dunes de sable font aujourd'hui partie du paysage à M'hamid, Ktaoua, Fezouata, voire même à Ternata en amont de Zagora. Elles envahissent les champs, enterrent les équipements y compris les canaux, les routes et les habitations, ce malgré l'effort fourni par les services techniques de l'État et la société civile.

Outre ces fléaux, en partie expliqués par les interventions maladroites des hommes, la vallée constitue le théâtre de la maladie du Bayoud (autre nom de la fusariose), qui ravage le palmier dattier ${ }^{(11)}$. La maladie est, selon les spécialistes, transmise par les eaux d'irrigation et par les sols. Elle attaque essentiellement les meilleures essences du palmier et, par conséquent, celles des dattes les mieux appréciées sur les marchés. Outre le Bayoud, le milieu oasien constitue un espace favorable pour le développement des sauterelles. Il a connu, durant son histoire, plusieurs épisodes, dont la dernière invasion en 2004. Ce phénomène saisonnier se renouvelle notamment lors des années fastes. Les acridiens, mais aussi les méthodes utilisées pour les éradiquer causent d'énormes dégâts à la flore et à la faune (usage massif du DDT).

\section{Les mutations socio-spatiales}

Parallèlement à cette situation écologique alarmante, la population a connu des mutations profondes essentiellement dues à la pression sur les ressources. L'accroissement quantitatif et qualitatif des besoins, dû en partie à la sédentarisation accélérée des nomades, à l'extension de l'urbanisation, à l'amélioration du niveau de vie et à l'orientation de la zone vers l'activité touristique, exerce une forte pression sur les ressources naturelles. À l'instar de ce qui se passe à l'échelle du Maroc, la population urbaine de la province de Zagora a triplé en trente ans, malgré son caractère encore rural (85 \% en 2004). En fait, la sédentarisation des nomades, l'exode rural, la promotion des centres, ont séduit les ruraux victimes des conditions naturelles ou qui cherchaient un emploi stable hors du secteur agricole. Le tourisme, longtemps concentré sur les zones côtières et les villes impériales du Nord, a trouvé dans les villes de Ouarzazte ${ }^{(12)}$, Zagora et Erfoud son paradis.

Les richesses patrimoniales, reliques de l'histoire, la beauté de la nature qu'offre la bande oasienne au milieu du désert, la clarté du ciel toute l'année, la netteté des couleurs et la nudité des paysages, l'architecture en pisé des ksour, les dunes de sable et la traditionnelle hospitalité des populations, attirent des foules de touristes nationaux et internationaux. 
Chance économique, le tourisme est aussi consommateur de l'eau, de l'espace et grand destructeur de l'environnement.

\section{La migration et l'environnement : quelle relation?}

La zone choisie pour notre enquête se situe en aval des palmeraies du Moyen Drâa. Elle se caractérise par une forte émigration et une importante dynamique environnementale. L'enquête, entreprise dans le cadre du projet EACH-FOR, a été menée auprès d'une soixantaine d'exploitants choisis selon leur statut migratoire (migrants et non-migrants) outre une dizaine d'entretiens auprès de responsables, de cadres et d'experts locaux choisis de façon strictement aléatoire.

L'analyse des résultats montre qu'environ 70 \% des répondants évoquent la mauvaise qualité de l'environnement comme cause de leur décision de migration.

Pour les migrants, la construction du barrage au début des années soixante-dix et la sécheresse des années quatre-vingt ${ }^{(13)}$ furent décisives. Elles ont renforcé une tradition migratoire déjà existante en ouvrant le chemin au regroupement familial. Au sein de ce groupe, trois sous-groupes se dégagent.

Tout d'abord, les cultivateurs oasiens qui possédaient, avant la migration, de la terre, de l'eau pour l'irrigation et quelques têtes de bétail à l'étable, mais qui vivent dans une situation de vulnérabilité critique. Ensuite, les éleveurs transhumants dont l'activité économique était étroitement liée aux précipitations et aux conditions climatiques. Avec les conflits frontaliers, leur mode de vie s'est complexifié par la brutale sédentarisation. Dans ce groupe, on rencontre les Aït Atta ${ }^{(14)}$, généralement sédentarisés pour se reconvertir en agriculteurs avant de repartir vers d'autres destinations, et les Aribs, qui, en s'installant à M'hamid, se sont reconvertis dans des activités non agricoles (commerce, tourisme, armée). Enfin, les Draouas, communauté noire, sans terres et sans cheptel, qui exerçaient chez les propriétaires terriens $\left(\right.$ Ahrars $\left.^{(15)}\right)$. Sans véritable attache au bled, ils sont les premiers à avoir émigré vers les villes, où ils achèvent de s'installer.

Ces émigrés, forcés, s'orientent essentiellement vers les grandes villes (Casablanca, Rabat, Kenitra, Agadir, Laayoun, Dakhla et Marrakech). Ils entretiennent généralement des relations privilégiées avec le bled où ils conservent de la famille et des biens. Aussi, les équipements récemment installés dans les zones de départ ne semblent guère pouvoir les retenir. Orientés essentiellement vers la consommation, ces équipements créent et augmentent les besoins en argent. L'argent est désormais le seul moyen d'échange, mais il n'est pas disponible sur place : il faut donc aller le chercher. 


\section{Des migrants qui vieillissent hors de chez eux}

Les caractéristiques sociodémographiques des migrants peuvent-elles expliquer la relation migration/environnement? Les émigrants sont en majorité de sexe masculin, mariés, âgés entre 48 et 58 ans et peu alphabétisés. C'est une tranche de population qui a, en partie, vieilli en dehors de chez elle.

Les femmes, si elles ne sont pas accompagnées, ne quittent presque jamais leur village où elles jouent un rôle socio-économique important. Le travail hors de leur foyer et de leur communauté est prohibé. Il est considéré comme une atteinte à leur dignité et à celle de leur famille et de leur clan. Aujourd'hui, avec la scolarisation, les femmes célibataires, veuves ou mariées, participent au mouvement migratoire et se valorisent de plus en plus en ville, voire même à l'étranger.

Dans une telle société, les enfants forment un capital socio-économique important. La quasi-totalité des émigrés a entre 4 et 6 enfants. Plus de 60 \% vivent encore avec leurs parents. La famille étendue reste fréquente. La taille moyenne des ménages est d'environ 8 personnes. L'analphabétisme touche près de 75 \% des émigrés.

Ces indices de précarité, face à la constante dégradation de l'environnement, entravent l'évolution socio-économique des émigrés et rendent leur exil interminable et leur intégration pénible. 


\section{Une stratégie d'attente chez ceux qui restent}

Face à ce contexte de dégradation, la résistance à la migration mérite une explication. $30 \%$ des répondants ont bien un projet migratoire, mais ils guettent une occasion qui tarde à venir pour partir à l'aventure. Interrogés à ce propos, les non-migrants évoquent une multitude de raisons nourries d'incertitudes et d'hésitation. Parmi les principales citées, on retrouve : l'existence d'une contrainte sociale ou physique qui empêche le répondant de quitter son village ; la pratique de la migration saisonnière comme solution temporaire ou le bénéfice de la rente migratoire d'un des siens ; la gestion d'une entreprise qui permet au répondant de récupérer l'argent de ceux qui partent tout en restant au bled; un antécédent migratoire décevant qui nourrit un comportement d'hésitation entre partir et rester.

Contrairement à la catégorie des migrants, les non-migrants présentent les traits d'une population jeune (30\% ont moins de 30 ans), relativement instruite (plus de $30 \%$ ont un niveau de secondaire), et célibataire (à $50 \%$ ). On rencontre aussi une cohorte de migrants retournés dans la zone à cause de leur âge ou après avoir installé leurs enfants ou monté une activité qui leur procure des revenus stables.

Aujourd'hui, rester au bled ne veut donc pas du tout dire renoncer au départ, mais plutôt penser au départ. Les départs causés par les risques de l'environnement, ceux stimulés de l'étranger, se font généralement sans préméditation. Le comportement démonstratif des émigrés nourrit le sentiment d'insatisfaction et l'espoir de partir chez les non-migrants.

\section{L'environnement oasien : perceptions croisées}

L'environnement est souvent évoqué comme une contrainte structurelle voire fatale avec laquelle on doit composer. Ainsi, pour les populations locales, la dégradation de l'environnement conditionne certainement la production agricole. La rareté de l'eau, l'érosion des sols, la salinisation, l'ensablement, la maladie du Bayoud, l'invasion des sauterelles et la régression des espaces pastoraux entravent la vie économique des populations. Ces facteurs, même s'ils avaient toujours existé ont changé d'ampleur depuis plusieurs décennies.

La marginalisation, le sous-équipement, l'enclavement, l'exclusion ainsi que les conflits locaux et régionaux ne sont réellement ressentis qu'avec la prise de conscience stimulée par le contact avec les autres sous ses différentes formes. Si l'accès à la terre, 
l'accès à l'eau et aux pâturages relevait jadis du statut social et de la capacité de les défendre, aujourd'hui, les conflits d'usage des ressources sont très ressentis (conflit avec les touristes et les urbains, conflits entre nomades et oasiens...).

Pour les paysans sédentaires, l'oasis était un vrai verger où l'on pouvait cultiver et réussir parfois trois récoltes par an et élever des animaux ; aujourd'hui, on ne peut plus rien y récolter. Pour les nomades, la dégradation de l'environnement se manifeste par le rétrécissement des espaces pastoraux et la disparition de leur couvert végétal.

La situation excentrique et frontalière des deux palmeraies (Ktaoua et M'hamid) augmente leur marginalité et leur isolement et, par conséquent, leur vulnérabilité socio-économique. L'accès à l'éducation, à la santé, aux éléments du confort devient le mot d'ordre de tous. L'école, les médias, la société civile, les touristes, les émigrés vulgarisent des valeurs qui font qu'on n'accepte plus, et de la même façon, les situations d'isolement, de marginalisation et d'exclusion. L'insatisfaction économique est donc une façon d'exprimer la pression de l'environnement dans sa dimension globale.

À l'unanimité, les répondants ont déclaré que la construction du barrage Mansour Eddahbi constituait un facteur de perturbation des conditions de production. Les palmeraies situées en aval (Ktaoua et M'hamid) ont vu leurs nappes asséchées et le taux de salinité des sols et des eaux augmenter. L'assèchement du lac d'Irqi a causé la perte de la faune et de la flore jadis alimentées par les crues de l'Oued Drâa. La régression des rendements, leur irrégularité, sont décisives pour plus de deux tiers des interviewés.

Ainsi, si le concept d'environnement n'apparaît pas clairement dans le discours, le recoupement des réponses montre qu'il est fortement présent dans la vie des populations. La transhumance, le comportement nataliste des populations et la migration forment autant de stratégies que la population utilise pour pallier la rareté, la fragilité des ressources et les risques environnementaux.

\section{L'avenir des oasis, de l'agriculture au tourisme}

Les propos tenus par les experts, les techniciens, les responsables, les élus et les membres de la société civile sont aussi instructifs même s'ils peuvent apparaître contradictoires. À propos de l'agonie prévisible des oasis, les uns l'imputent à l'inadéquation des interventions de l'État et à l'effritement des systèmes traditionnels de gestion, alors que les autres incriminent la mauvaise gestion des ressources : sur- 
pompage, morcellement des terres, complexité des statuts fonciers et surpeuplement. L'aménagement hydro-agricole de la vallée du Drâa, a perturbé le système écologique et socio-économique hérité, sans innover pour valoriser et améliorer les conditions de vie des populations. Il faut que les populations se mobilisent pour valoriser et capitaliser les actions des autres. Alors que pour le technicien, "c'est la population qui est incapable d'apprécier les efforts de l'État'.

Le changement du climat, la rareté de l'eau, la dégradation du couvert végétal et des sols, face au surpeuplement des oasis et l'explosion des besoins, poussent les gens à émigrer. "Nous ne pouvons plus rétablir la situation et empêcher les hommes d'émigrer. Il faut

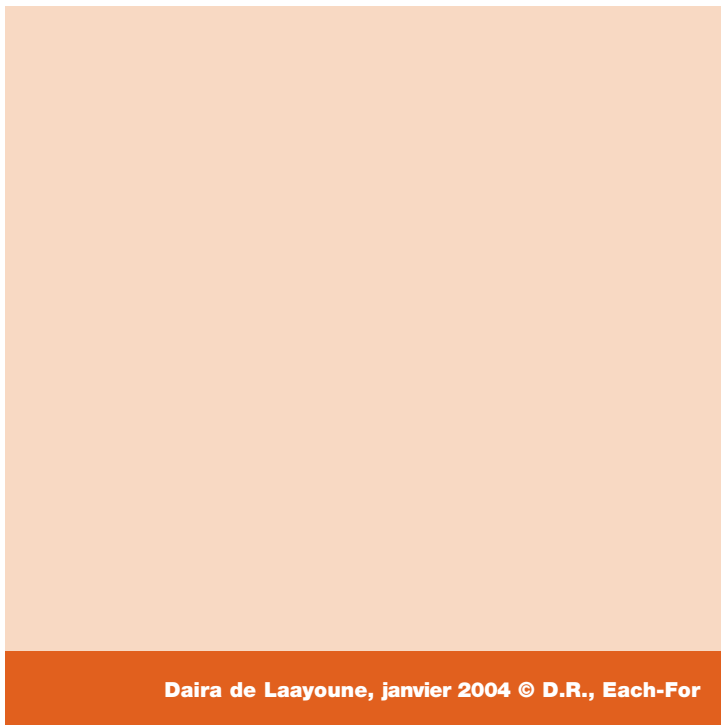
juste maintenir l'équilibre minimum en recourant à d'autres activités telles que le tourisme et le cinéma, afin de garantir de l'emploi aux gens. Il faut déclarer la fin de l'ère agricole et penser tourisme et cinéma", précise un élu. Les problèmes environnementaux dépassent largement les moyens et les compétences des locaux, qui, avec la collaboration d'autres partenaires, tentent d'en atténuer les effets. La grande ambition est de faire de ces zones des destinations touristiques attractives pouvant assurer de l'emploi aux jeunes, afin d'y insuffler une nouvelle dynamique tout en préservant les systèmes écologiques.

Reste à imaginer quel attrait auront les oasis sans eau, sans agriculture et sans les oasiens? Les touristes et les gens du cinéma auront-ils la capacité et le savoir-faire pour gérer la fragilité et maintenir cet héritage millénaire?

\section{Conclusion}

Pour clore, nous pouvons dire que l'étude était une occasion de vérifier la relation supposée entre l'environnement et la migration. Or dans notre cas, l'austérité naturelle est une donnée structurelle. 
Aussi, nous devons rappeler que la migration est un fait social naturel, et que seules les grandes vagues de population, stimulées ou attirées par des conjonctures exceptionnelles, sont à discuter. La question de ceux qui décident de rester même si les conditions sont défavorables et de ceux qui partent, alors qu'ils vivent dans l'aisance, rend les réponses très discutables. Aussi, l'attachement généralement manifesté par les émigrés envers leur pays d'origine, malgré l'âpreté des conditions naturelles, laisse dire que la question de la migration est très complexe.

Les grandes ruptures environnementales imposent, certes, le départ des populations, mais elles ne peuvent pas en être l'unique cause. Elles peuvent même dans des cas stimuler des adaptations et éveiller le génie humain. L'environnement socioculturel, économique et politique a une égale importance dans la prise décision pour partir ou rester.

\section{Bibliographie}

- M. Ait Hamza, Mobilité socio-spatiale et développement local au sud de l'Atlas marocain (Dadès-Todgha),

Maghreb Studien 13, Passau, 2002, 196 p.

- M. Aït Hamza., "L'émigration, facteur d'intégration ou de désintégration des régions d'origine ? Cas du bassin versant d'Assif Mgoun", in Le Maroc et la Hollande. Études sur l'histoire, la migration, la linguistique et la sociologie de la culture, Rabat, FLSH, Série Colloques et Séminaires, n8, 1988, pp.161-175.

- M. Aït Hamza, "Migration et dynamique de l'espace local : Bouteghrar (versant sud du Haut Atlas central)", in Berriane \& H. Popp (éd.), Migration internationale entre le Maghreb et l'Europe, Passau, Maghreb-Studien n 10, 1998, pp. 147-158.

- A. Bahani, Les structures agraires, les systèmes d'irrigation (palmeraie de Fezouata, Draâ Moyen, Maroc), Thèse de géographie, 2 T, Université Rouen (ronéo), 1990.

- A. Bencherifa \& H. Popp, Loasis de Figuig : persistance et changement, Passau, Mittelmeerstudereihe, n², 1990.

- BENTALEB Aziz, 2008, La dynamique de désertification dans le bassin du Drâa moyen : analyse et perspectives. Thèse de doctorat national en géographie, Université Mohammed V, Agdal, Faculté des Lettres et des Sciences Humaines, Rabat (ronéotypé).

- Ch. De Foucauld (Vicomte), Reconnaissance au Maroc, Paris, Edition Challamel, 1888.

- Djerbi et al., "Preliminary Results of Selection of High-Quality Bayoud-Resistant Clones Among Natural Date Palm Population in Morocco", in Processing of the Second Symposium on the Date Palm, Saudi Arabia, 1986, pp. 383-399.

- W. F. Heinmeyer et al., Partir pour rester. Enquête sur les incidences de l'émigration ouvrière à la campagne marocaine, La Haye, Publication de l'Institut sociogéographique de l'Université d'Amsterdam, 1977.

- Direction de la Statistique (éd.), Recensement général de la population et de l'habitat 1971 : Population rurale. Région Sud, Rabat, Imprimerie Casablanca, 1973. 
- Direction de la Statistique (éd.), La population légale du Maroc selon le Recensement de 1982, Rabat, Imprimerie Casablanca, 1983

- Direction de la Statistique (éd.), Recensement général de la population et de l'habitat selon de 1982 : Population rurale : Région du Sud, Rabat, Imprimerie Casablanca, 1984.

- Direction de la Statistique (éd.), Recensement général de la population et de l'habitat de 1994 : Population rurale par fraction et douar, Rabat, Imprimerie Casablanca, 1996.

- L. Ouhajou, Espace hydraulique et société au Maroc. Cas des systèmes d'irrigation dans la vallée du Drâa, Agadir, Faculté des lettres et des sciences humaines, Thèse et Mémoire nº 7,1996.

- L. Ouhajou, Étude des structures socio-spatiales des groupes cibles. Projet de lutte contre la désertification dans la vallée du Drâa (Rapport, ronéo, ORMVAO, /GTZ), 1993.

- A. T.Zainabi, Mouvement associatif moderne et développement local. Cas de la province de Zagora (thèse en cours sous la direction de M. Ait Hamza), UFR Aménagement et Développement rural au Maghreb (FLSH), Univ. Mohammed V, Agdal, Rabat.

- Haut Commissariat au Plan, RGPH 2004, www.hcp.ma.

\section{Notes}

1. La "palmeraie de Ktaoua" est appelée comme telle ou par le nom de la commune de Tagounite. Les deux termes sont utilisés dans ce rapport. Le Centre rural de Tagounite, siège de la commune, n'est apparu que lors du dernier découpage administratif de 1992. M'hamid est communément appelé "M'hamid El Ghizlane" (des gazelles), mais dans le texte, on utilisera souvent le nom "M'hamid".

2. "Draouas" : dérivé du mot "Dra". Le mot signifie les populations qui habitent la vallée du Drâa. Mais par extension, le concept désigne des populations noires, probablement d'origine saharienne ou subsaharienne, ayant généralement un statut d'homme attaché à la terre agricole, mais sans terre.

3. La tribu des Aït Sedrate est une tribu makhzeniènne qui, sur appel des Zaouia Idrissides, a migré vers le sud et arrive vers le Dades puis dans le Drâa pour protéger les Draouas.

4. Les Aït Atta forment une tribu ou confédération de tribus essentiellement nomade(s). Arrivées du Sahara et au contact des autres tribus, les Aït Atta se sont constitués en confédération sur un territoire qui s'étend jusqu'au Tafilalet, au Haut Atlas, voire même jusqu'aux plaines de Tadla et du Haouz au nord de l'Atlas.

5. En 1936, les deux palmeraies abritaient 1169 juifs.

6. Ces termes doivent être compris dans leur sens matériel mais aussi moral.

7. L'indice de fécondité dans les deux palmeraies a atteint 3,4 à Ktaoua et 2,9 à M'hamid (2004).

8. Le henné est une plante aromatique, médicinale très exigeante en matière d'irrigation et d'entretien. Son extension dans la zone est liée au pompage. On la rencontre surtout dans la palmeraie de Mezguita, Tinzouline, et hors de la vallée dans la zone de Tazarine.

9. Les grands rameaux de la confédération Aït Atta qu'on rencontre dans les deux palmeraies sont les Aït Boudaoud et les Aït Isfoul.

10. La capacité de stockage du barrage est de 560 millions de $\mathrm{m} 3$ d'eau. Néanmoins et depuis sa construction, rares sont les années où celui-ci a dépassé $60 \%$ de sa capacité.

11. L'enquête menée par Djerbi en 1981 a révélé que le Bayoud a tué dans la vallée environ 165574 pieds sur les 2 millions au total.

12. Ouarzazate se considère déjà, avec ses nombreux studios, comme la capitale du cinéma au Maroc.

13. Les conséquences de l'arrêt de l'eau derrière le barrage coïncidaient avec cette sévère sécheresse. Ce qui a accéléré la dégradation des écosystèmes oasiens du Drâa et notamment ceux de l'aval ou se situent nos sites d'enquêtes.

14. Leur installation s'est traduite par des extensions d'oasis par pompage et par conséquent, un appauvrissement de la nappe.

15. "Ahrar" : "les libres". 